\title{
Effect of Polyethers for Photo and Thermal Decomposition of Photosensitive Diazo Compounds
}

\author{
Kieko Harada ${ }^{1}$, Tetsuyuki Taniai ${ }^{1}$, Masahiro Nakada ${ }^{1}$, Hiroshi Hamana ${ }^{2}$ and Kazuyuki Sugita ${ }^{3}$ \\ 1 Education Center, Chiba Institute of Technology, 2-1-1 Shibazono, Narashino-shi, Chiba 275-0023 Japan \\ 2 Faculty of Engineering, Saitama Institute of Technology, 1690 Fusaiji, Fukaya-shi, Saitama 369-0293, \\ $J A P A N$ \\ 3 Faculty of Engineering, Chiba University, 1-33, Yayoi-cho, Inage-ku, Chiba 263-8522 Japan
}

\begin{abstract}
Inclusion compound formation of polyethylene glycol (PEG) with p-substituted benzene diazo compounds (SBD) in aqueous solutions was decided from activation energy (Ea) of thermal decomposition, dissociation constant $\mathrm{Kd}$ by Lineweaver-Burk plot modified with Michaelis-Menten formula and degradation temperature $\mathrm{Dt}$, and compared with constitution of complex by WinMOPAC calculation. Thermal decomposition rate of p-chlorobenzene diazo compound (CIBD) with PEG was accelerated as with $\beta$ cyclodextrine (CD) which include the benzene ring of the diazo compound. $\mathrm{Kd}$ of CIBD with PEG was larger than that with $\mathrm{CD}$. PEG is not a ring, but a linear molecule. Dt of SBD with PEG in solid was higher than that without PEG300. Effect of molecular weight of PEG for Dt was, in a decreasing order, no additives $\sim$ PEG $300<$ PEG600 < PEG $1000 \sim$ PEG 2000. Thermal decomposition of a diphenylamine-4-diazonium sulfate salt/formaldehyde condensate (DSR) / Polyvinyl alcohol (PVA) resist with PEG was accelerated. Photo decomposition of DSR/PVA resist with PEG 300 and 600 was also accelerated. Benzene diazo compound with PEG 300 was more stable than neat BD from by WinMOPAC caluculation.
\end{abstract}

Keywords: photosensitive diazo compound, 18-crown-6, polyethylene glycol 300, $\beta$ cyclodextrin,

\section{Introduction}

The para-substituted benzene diazo compounds (SBD) with electron-donating groups (DSBD) are used as the photoacid generator for micro resists [1], photosensitive emulsions for screen printing resists [2], and UV-fixable thermal recording papers [3-5]. SBD with electron withdrawing substituted (WSBD) were used for acid generators for cationic polymerization. 18-Crown-6 (18C6) complexes with benzene diazonium cation improve photo and thermal stability of diazo compounds [6-11]. Thermal decomposition of less expensive $\beta$-cyclodextrin (CD) complexes with diphenylamine-4-diazonium sulfate/formaldehyde condensate / PVA resist (DSR-PVA) was suppressed [11]. DSBD with CD will be improved thermal stability without decreasing sensitivity [12]. The coloring of the DSR/PVA emulsion films was suppressed by inclusion of $18 \mathrm{C} 6$ or PEG [11].

In this report, inclusion compound formation of polyethylene glycol (PEG) with p-substituted benzene diazocompounds (SBD) in aqueous solutions was decided from activation energy (Ea) of thermal decomposition, dissociation constant $\mathrm{Kd}$ by Lineweaver-Burk plot modified with MichaelisMenten formula, and degradation temperature Dt. and compared with constitution of complex by WinMOPAC caluculation. Application of PEG to DSR / PVA resist was studied by measurment of thermal and photo decomposition.

\section{Experimental}

2.1 Materials

p-Substituted benzene diazonium tetrafluoroborates (SBD) were precipitated by adding hydrogen tetrafluoroborate to an aqueous solution of the corresponding benzene diazonium chloride prepared from $p$-substituted aniline. The solid product was recrystallized from ethanol. The conventional DSR photosensitive diazo resin for screen platemaking and PVA emulsion for screen printing were

Received March 31, 2007

Accepted May 10, 2007 
purchased from Murakami Screen K.K. The additives used were, PEG300, PEG600, PEG1000, PEG2000 and CD of extra pure grade.

Concentrations of SBD and inclusion compounds in an aqueous solution were $2.5 \times 10^{-4} \mathrm{~mol} / \mathrm{l}$, $1.25 \times 10^{-3} \sim 1.25 \times 10^{-2} \mathrm{~mol} / 1$, respectively. Solid for thermal analysis was formed by dissolving SBD in liquid PEG.300 or melted SBD with PEG 1000 or PEG2000.

\subsection{Measurements}

Thermal decomposition of SBD in an aqueous solution was monitored by measuring the UV absorption intensity at $\lambda$ max under light-shielded conditions. Photo decomposition of SBD in an aqueous solution was conducted by irradiation with a Toshiba Super High-Pressure Mercury lamp SHL100. The incident energy was $3.1 \mathrm{~mW} / \mathrm{cm}^{2}$. The absorbances at $\lambda$ max of UV spectra were measured with a Shimazu UV-1600 PC spectrophotometer. Thermal degradation temperature was determined by TDA and TGA in solid measured with a Shimazu ETG-60 Analyzer.

\subsection{Computational experiment}

Calculation was performed with WinMOPAC ver. 3.5.1a (served by Fujitsu Co. Ltd.) in a personal computer. This software combined a graphical user interface for Z-matrix and MOPAC2002 [13] for calculating engine. The EF routine and MOZYME [13] routine were used for optimizer. The Parametric Method 5 (PM5) was used. For other parameters default values programmed in MOPAC2002 were used.

In order to build a structure of PEG600, a molecular dynamics simulation program of Chem3D Pro ver.10.0 (served by CambridgeSoft ) was used. The obtained structure at $300 \mathrm{~K}$ was optimized with MOZYME routine of MOPAC 2002 .

\section{Results and Discussion}

3.1 Inclusion compound formation

3.1.1 Thermal decomposition

Thermal decomposition reaction is as follows:

$\mathrm{k} 1$ k2

$\mathrm{P} 1 \leftarrow \mathrm{D}+\mathrm{I}=\mathrm{D} \cdot \mathrm{I} \rightarrow \mathrm{P} 2+\mathrm{I}$

\section{D : Diazocompound \\ I : Inclusion compound \\ P : Product}

Dissociation constant $\mathrm{Kd}=[\mathrm{D}][\mathrm{I}] /[\mathrm{D} \cdot \mathrm{I}]$
Overall rate of decomposition $\mathrm{V}$

$$
\begin{aligned}
\mathrm{V} & =\mathrm{k} 1[\mathrm{D}]+\mathrm{k} 2[\mathrm{D} \cdot \mathrm{I}] \\
& =\mathrm{k} 1[\mathrm{D}]+\mathrm{k} 2[\mathrm{D}][\mathrm{I}] / \mathrm{Kd} \\
& =(\mathrm{k} 1+\mathrm{k} 2[\mathrm{I}] / \mathrm{Kd})[\mathrm{D}]
\end{aligned}
$$

$\mathrm{k} 1$ : rate constant of free (dissociated) D

k2 : rate constant of complexed D

Apparent rate constants of decomposition of $D$ in the absence and presence of $I$ are defined as kun and kobs, respectively.

$\mathrm{V}=\operatorname{kun}[\mathrm{D}]=\mathrm{k} 1[\mathrm{D}]$

Net rate constant of complexed D, kobs-kun

Lineweaver-burk plot modified with MichaelisMenten formula $[14,15]$ is shown as follow

$$
1 /(\text { kobs-kun })=\mathrm{Kd} / \mathrm{k} 2 \cdot 1 /[\mathrm{I}]+1 / \mathrm{k} 2
$$

Para-chlorobenzene diazo compound (ClBD) $\left(2.5 \times 10^{-4} \mathrm{~mol} / \mathrm{l}\right)$ complex formed by adding $1.25 \times 10^{-3}$ and $1.25 \times 10^{-2} \mathrm{~mol} / 1$ ( 5 and 50 times of ClBD ) of PEG300 was thermally decomposed in an aqueous solution. After thermal decomposition, remainng free $\mathrm{CIBD}$ and complexed $\mathrm{ClBD}$ was

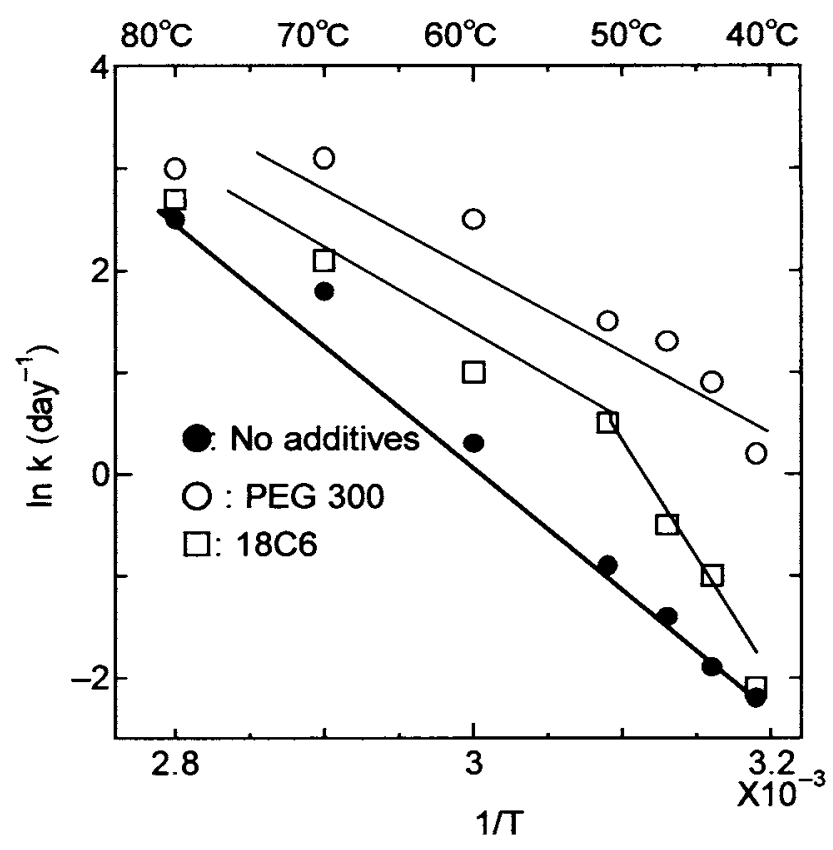

Fig.1 Arrhenius plots of the rate constants of the neat $\mathrm{CIBD}$ and CIBD complexes with PEG300 or 18C6 : : $\mathrm{ClBD}\left(2.5 \times 10^{-4} \mathrm{~mol} / \mathrm{l}\right), \mathrm{O}: \mathrm{CIBD}+\mathrm{PEG}$ $300\left(1.25 \times 10^{-2} \mathrm{~mol} / \mathrm{l}\right), \square: \mathrm{ClBD}+18 \mathrm{C} 6\left(1.25 \times 10^{-2}\right.$ $\mathrm{mol} / \mathrm{l})$

determined photochemically by measuring the intensity of maximum absorption band at $283 \mathrm{~nm}$. 
Thermal decomposition of neat (or free) ClBD and complexed CIBD with PEG in aqueous solutions was first-order decomposition.

The plots of the rate constants of CIBD $(\mathrm{kun}=\mathrm{k} 1)$ and complexed ClBD (kobs) with 50 times of PEG 300 versus $1 / \mathrm{T}$ (Arrhenius plot) are shown in Fig.1.

The plot of 1:50 (molar ratio) showed a straight line, though there was observed a diviation from the line on the plot of the 1:5 PEG complex. In order to compare with 1:50 18C6 complexes, the Arrhenius plot of $18 \mathrm{C} 6$ complex is also shown in Fig.1. There is a bending point of the plots of 1:50 $18 \mathrm{C} 6$ complexes of CIBD. It was reported, there is the bending point of a plots of $18 \mathrm{C} 6$ complexes of benzene diazo compound in methanol [7]. ClBD dissociates from $18 \mathrm{C} 6$ above $48^{\circ} \mathrm{C}$, which causes the diazonium moiety free from the ligand through the entropic effect [7].

Activation energies (Ea) were obtained from the plots and are shown in Table 1.

Table 1. Activation Energy (Ea) and dissociation constant (Kd) of p-chlorobenzene diazonium (CIBD) and PEG - or 18C6-complexed CIBD in an aqueous solution

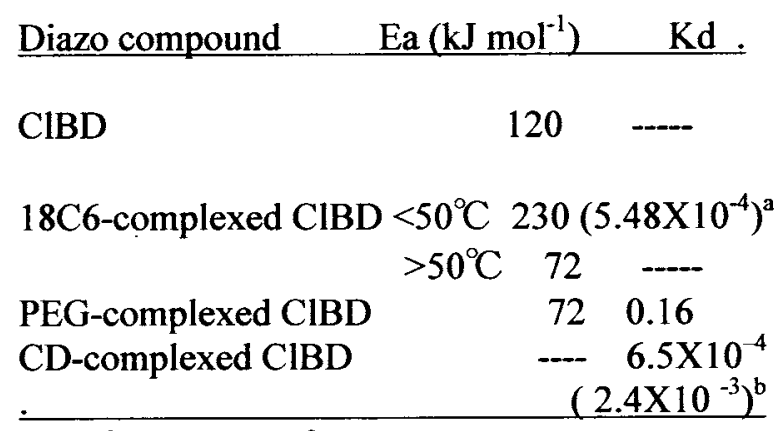

$\mathrm{a}: 50^{\circ} \mathrm{C}[6], \mathrm{b}: 26^{\circ} \mathrm{C}[15]$

Ea's of PEG-complexed ClBD and 18C6complexed CIBD (above $50{ }^{\circ} \mathrm{C}$ ) were $72 \mathrm{KJ} \mathrm{mol}^{-1}$. Rate constant, $k$, of the former compound was larger. Thermal decomposition of CIBD was accelerated, when complexed with PEG containing hydroxy groups at the chain ends. The thermal and photo decompositions of diazocompound were not accelerated by the PEG with methoxy end-groups [16]. PEG includes the benzene ring of diazo compound similarly as $\mathrm{CD}$.

Ea of 18C6-complexed CIBD (below $50{ }^{\circ} \mathrm{C}$ ) was $230 \mathrm{KJ} \mathrm{mol}^{-1}$ and larger than that of neat $\mathrm{ClBD}, 120$
$\mathrm{KJ} \mathrm{mol}^{-1}$. Since $18 \mathrm{C} 6$ includes the diazo group of diazo compound below $50{ }^{\circ} \mathrm{C}[7]$, the thermal decomposition was suppressed.

\subsubsection{Dissociation constant}

Dependence of net decomposition rate constant of the complex, kobs-kun, on the concentration of PEG300 used as the inclusion compounds is shown in Fig.2. Dissociation constant (Kd) of ClBD complex with PEG300 was decided by LineweaverBurk plots modified with Michaelis-Menten formula shown in Equation (2).

CIBD $\left(9.0 \times 10^{-4} \mathrm{~mol} / \mathrm{l}\right)$ with PEG300 (5 10 times ClBD) or with $\mathrm{CD}(0.5 \sim 10$ times) were thermally decomposed at $40^{\circ} \mathrm{C}$.

Plots of the net rate constant (kobs-kun) against additive concentrations of PEG was found to show a saturation phenomena. The reciprocal of net rate constants is linearly proportional concentration of additive concentration of PEG300 as shown in Fig.3.

From this result, it was considered that PEG formed a complex with CIBD.

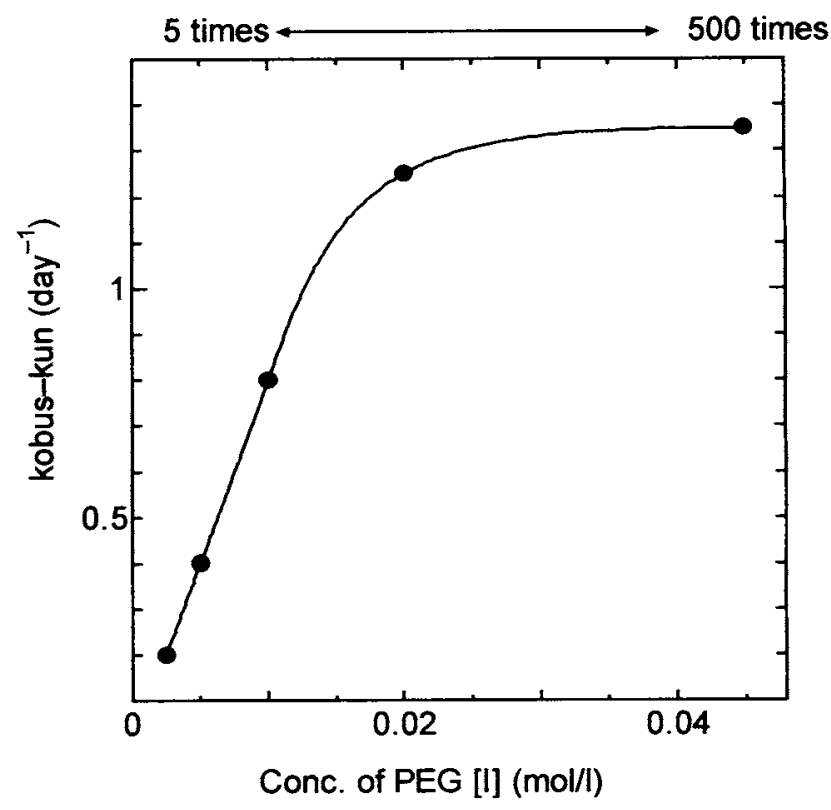

Fig.2 Dependence of decomposition rate constant on the concentration of PEG300 [ ClBD : 9X10 ${ }^{-4}$ mol/l aqueous solution] $40^{\circ} \mathrm{C}$ 


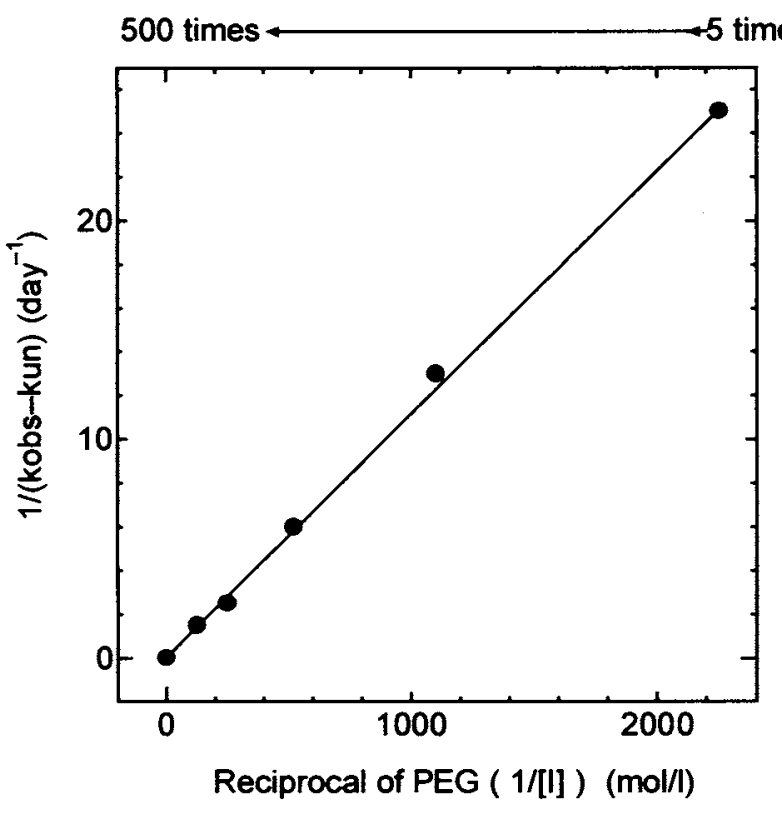

Fig, 3 The relation of the reciprocal of net rate constant of ClBD complex and the reciprocal of PEG300 concentration

Dissociation constants, $\mathrm{Kd}$, was decided from the slope of the the plots and is shown in Table 1. Kd of CD-complexed CIBD is shown in Table $1 . \mathrm{Kd}$ of PEG 300 was larger than that of CD. PEG 300 is not a ring, but a flexible linear molecule, so the PEG-complexed CIBD in an aqueous solution was unstable.

\subsubsection{Degradation temperature of solid complex}

Degradation temperature, Dt, of SBD complexes with PEG of various molecular weights was determined by TDA and TGA measured in solid. Molecular-weight effects of PEG on Dt of the complexes of benzene diazo compound (BD), ClBD and DSR are shown in Fig. 4.

Dt's of BD and ClBD with PEG 300 were lower than those without PEG300, respectively. Dt's of DSR with PEG were higher than that without PEG, in an increasing order : no additives $\sim$ PEG $300<$ PEG600 PEG1000 PEG 2000. Plots of Dt against molecular weight of $\mathrm{BD}$ and $\mathrm{DSR}$ were found saturations over molecular weight 600 .

PEG600, PEG1000 and PEG 2000 were solid at room temperature. When $\mathrm{BD}$ and DSR are included by PEG with molecular weight over 600 , they are stable. PEG300 with 6-10 oxygens simi- larly as $18 \mathrm{C6}$, is liquid and hygroscopic.

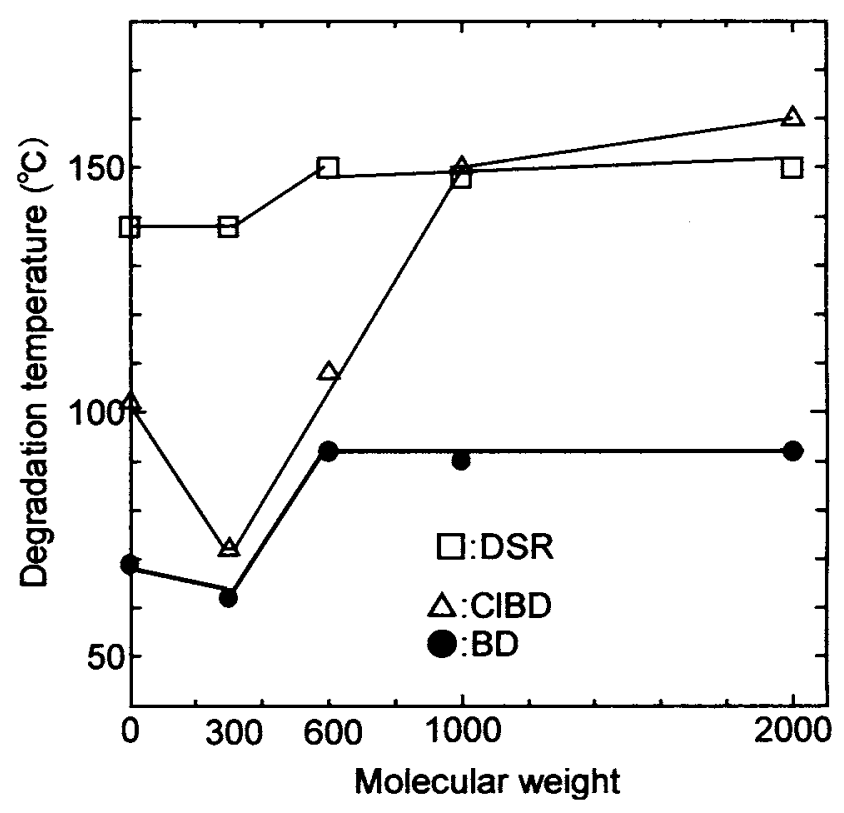

Fig. 4 Molecular weight effects of PEG on Dt of PEG-complexes SBD

Diazo compounds are thermally decomposed easily in aqueous solutions, and it is considered that diazo compounds are unstable in presence of hygroscopic PEG300.

3.1.4 Estimation of the constitution of the complex by WinMOPAC calculation

Optimized structures and the heat of formation of PEG300, PEG600, BD and the complex were calculated with WinMOPAC. Calculated heat of formation of BD, PEG300, PEG600 were 1038, $-1213,-2434 \mathrm{~kJ} / \mathrm{mol}$, respectively. The heat of formation of PEG300- complexed $\mathrm{BD}$ and PEG600- complexed BD were -308 and -1492 $\mathrm{kJ} / \mathrm{mol}$, respectively. Optimized structure of PEG300-complexed BD and PEG600- complexed BD were shown in Fig.5.

The heat of formation of PEG-complexed BD was smaller than the sum of the heat of formation of starting materials. The stabilization energy of PEG300-complexed BD and PEG600-complexed BD were estimated -133 and $-96 \mathrm{~kJ} / \mathrm{mol}$, respectively. These results support the formation of in- 
clusion compound of PEG with BD.

The calculated optimized structure of PEGcomplexed BD suggests, when molecular weight of PEG increases, the influence of hydroxyl end group of PEG becomes less predominantly. Therefore Dt of PEG600-complexed BD is higher than that of PEG300-complexed BD.
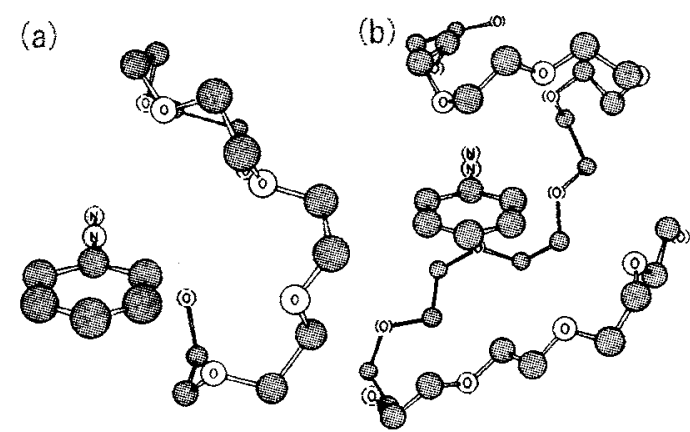

Fig. 5 Optimized structure of PEG- complexed $\mathrm{BD}$;(a): PEG300- complexed BD, (b): PEG600complexed BD

\subsection{Application of PEG for Diazo/PVA resist}

The PVA films containing diphenylamine-4diazonium sulfate /formaldehyde condensate (DSR) and PEG (thickness $250 \mu \mathrm{m}$ ) were stored at $50{ }^{\circ} \mathrm{C}$ in the dark, and absorbance at $\lambda \max (380 \mathrm{~nm})$ of UV spectra was measured every $24 \mathrm{hr}$.

The thermal decomposition of DSR with PEG of the solid films were studied as shown in Fig.6.

The relative thermal decomposition rate of the films was, in a decreasing order; PEG300 > PEG $600>$ PEG1000 > PEG $2000 \sim$ no additives, and corresponded directly to the reciprocal of molecular weight.

Although DSR with PEG600 was shown to be stable in solid by the experimental data of Dt, the DSR/PVA film was unstable PVA by hygroscopic PEG. for long storage. As thermal decomposition of diazo compounds is ionic reaction, it was accelerated by hygroscopic PVA.

The DSR/PVA films with or without PEG were irradiated with a mercury lamp, and the absorbance at $\lambda \max$ of UV spectra was measured every min. The photodecomposition of the films is shown in Fig. 7.

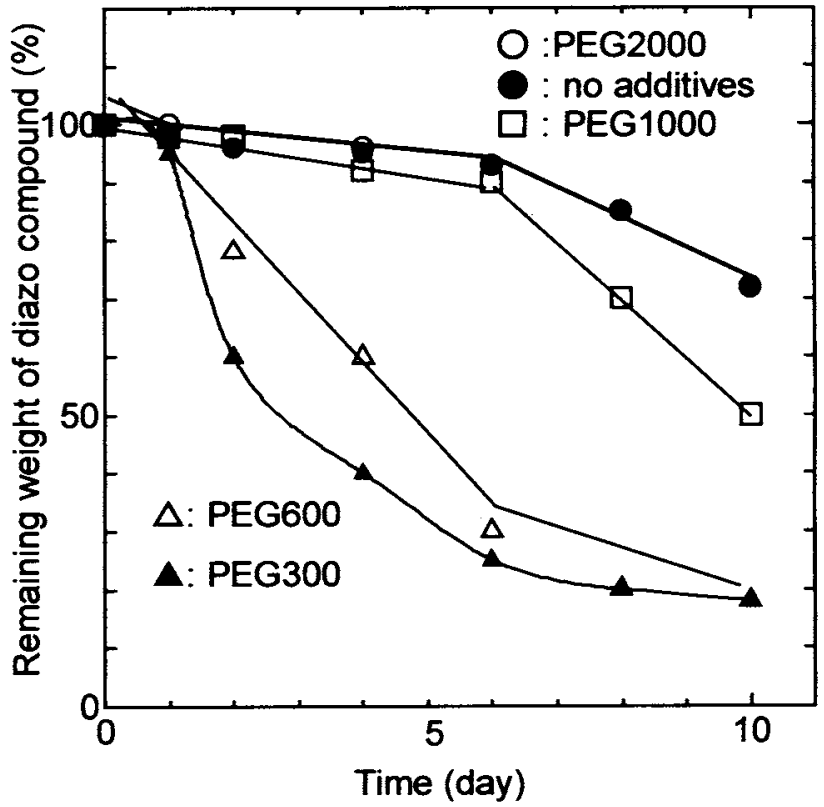

Fig.6 The thermal decomposition of DSR/PVA film with PEG Temperature:50 ${ }^{\circ} \mathrm{C}$. Thickness $250 \mu \mathrm{m}$

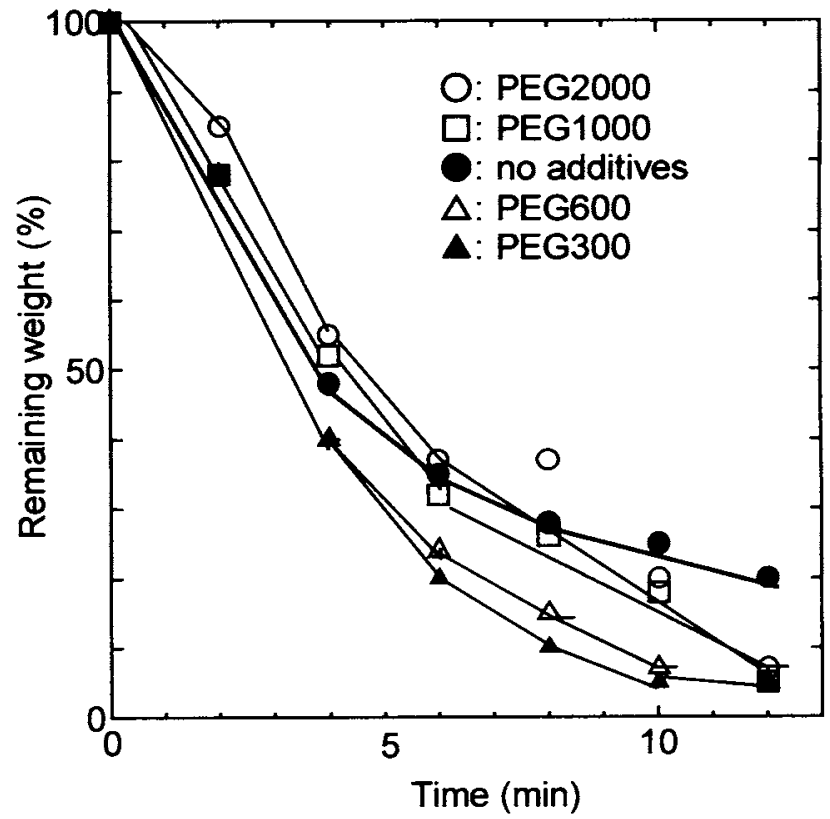

Fig.7 Photodecomposition of DSR/PVA films with PEG

The relative photodecomposition rates of the DSR/PVA were, in a decreasing order : PEG300 > PEG600 > PEG1000 > no additives PEG 2000. DSR/PVA films with PEG were accelerated except 


\section{PEG2000.}

The diazo group is bonded with hydroxy group of PEG like a diazo group bonded with a hydroxy group of CD [15]. The diazo included with PEG generates nitrogen gas and an alkoxy and a phenyl radicals via homolysis by irradiation. The homolysis is accelerated by formation of loose complex with PEG [16].

\section{Conclusion}

PEG300 formed with CIBD a weak complex in aqueous solution. Degradation temperatures of SBD with PEG in solid were higher than that without PEG. The DSR/PVA films with PEG 300 did not show long shelflife, though they showed high sensitivity.

\section{Acknowledgements}

The authors thank to Prof. Kyoichi Saito, Assoc. Prof. Masahito Kushida, and Mr. Hideki Ogasawara of Chiba University for their helpful suggestions.

\section{References}

1. K.Harada, M.Kushida, K.Saito, K.Sugita and H.Iida, "Micro- and Nanopattering Polymers" H.Ito, E.Reichmanis, O.Naramasu and T.Ueno, eds., ACS Symp. Ser.706, Amer. Chem. Soc., Washington, D.C., 1998, pp. 126-133

2. K.Harada, M.Kushida, K.Saito, K.Sugita and H.Iida, J. Imaging Sci. and Technol., 46, 159 (2002)

3. T.Usami, T.Tanaka and S.Ishige, Electrophotography, 26, 115 (1987)

4. Y.Yoshida and S.Tatsuta, Electrophotography, 26,120
5. R.A.Bartsch, H.Chen, N.F.Haddoch and P.N.Juri, J. Am. Chem. Soc., 98, 6753 (1976)

6. T.Kuokkanen and P.O.I.Virtanen, Acta Chem. Scand., ser. B, 33, 725 (1976)

7. K.Harada, M.Kushida and K.Sugita, J. Soc.

Photogr. Sci. Technol. Japan, 46,600 (1993)

8. K.Harada, K.Sugita and H.Iida, J.Imaging Sci. and Technol., 38, 13 (1994)

9. H.Nakazumi, I.Szele, K.Yoshida and H. Zollinger, Helvetica Chimica ACTA, 66, 1721 Y.Yoshida and S. Tatsuta, Electrophotography, 26, 120 (1987)

10. H.Nakazumi, T. Kitao and H.Zollinger, J. Organic Chem. 52, 2828 (1987)

11. K.Harada, M.Kushida, S.Miyagawa, S.Takahara and K.Sugita, J.Photopolym.Sci.Technol., 18, 187 (2005)

12. K.Harada, W. Aimaiti, M.Nakada, Y. Morihiro, T.Narita, R.Aoki.M.Kushida and K.Sugita, J.Photopolymer. Sci. Technol., 19, 129 (2006)

13. J. J. P. Stewart, Int. J.Quant.Chem., 58, 133 (1996)

14. H,Lineweaver and D.Burk, J.Am.Chem.,Soc., 56, 658 (1934)

15. K.Fukunishi, J.Hira, H.Yamaoka and M.

Nomura, J. Chem. Soc. Perkin Trans. I, p.991 (1985)

16. K.Harada, M.Kushida, K.Saito and K.Sugita, Advances in Imaging Materials and Process, (Proc.of $13^{\text {th }}$ Inter'I Conf. on Photopolymers; H.Ito, P.Rao Varanasi , M.M. Kojasteh and R.Chen, Eds., Soc. Plastics Eng. Inc., Mid Hudson Section, Hopewell Junction, NY,.2003) pp.369-373 